\title{
Long term gluten consumption in adults without celiac disease and risk of coronary heart disease: prospective cohort study
}

\author{
Benjamin Lebwohl, ${ }^{1,2}$ Yin Cao, ${ }^{3,4,5}$ Geng Zong, ${ }^{5}$ Frank B Hu, ${ }^{5,6}$ Peter H R Green, ${ }^{1}$ Alfred I Neugut, ${ }^{1,2}$ \\ Eric B Rimm,5,6,7 Laura Sampson, ${ }^{5}$ Lauren W Dougherty, ${ }^{5}$ Edward Giovannucci, ${ }^{5,6,7}$ Walter C Willett, 5,6,7 \\ Qi Sun, ${ }^{5,6}$ Andrew T Chan 3,4,6
}

${ }^{1}$ Celiac Disease Center, Department of Medicine, Columbia University College of Physicians and Surgeons, New York, NY, USA

2Department of Epidemiology, Mailman School of Public Health, Columbia University, New York, NY, USA

${ }^{3}$ Clinical and Translational Epidemiology Unit,

Massachusetts General Hospital and Harvard Medical School,

Boston, MA, USA

${ }^{4}$ Division of Gastroenterology, Massachusetts General Hospital and Harvard Medical School,

Boston, MA, USA

5Department of Nutrition, Harvard T. H. Chan School of Public Health, Boston, MA, USA

${ }^{6}$ Channing Division of Network Medicine, Department of

Medicine, Brigham and

Women's Hospital and Harvard

Medical School, Boston, MA,

USA

7Department of Epidemiology, Harvard T.H. Chan School of Public Health, Boston, MA, USA

Correspondence to: A T Chan ACHAN@mgh.harvard.edu

Additional material is published online only. To view please visit the journal online.

Cite this as: BMJ 2017;357:j1892 http://dx.doi.org/10.1136/bmj.j1892

Accepted: 4 April 2017

\section{ABSTRACT}

OBJECTIVE

To examine the association of long term intake of gluten with the development of incident coronary heart disease.

DESIGN

Prospective cohort study.

\section{SETTING AND PARTICIPANTS}

64714 women in the Nurses' Health Study and 45303

men in the Health Professionals Follow-up Study without a history of coronary heart disease who completed a 131 item semiquantitative food frequency questionnaire in 1986 that was updated every four years through 2010 .

\section{EXPOSURE}

Consumption of gluten, estimated from food frequency questionnaires.

\section{MAIN OUTCOME MEASURE}

Development of coronary heart disease (fatal or non-fatal myocardial infarction).

\section{RESULTS}

During 26 years of follow-up encompassing 2273931 person years, 2431 women and 4098 men developed coronary heart disease. Compared with participants in the lowest fifth of gluten intake, who had a coronary heart disease incidence rate of 352 per 100000 person years, those in the highest fifth had a rate of 277 events per 100000 person years, leading to an unadjusted rate difference of 75 (95\% confidence interval 51 to 98) fewer cases of coronary heart disease per 100000 person years. After adjustment for known risk factors, participants in the highest fifth of estimated gluten intake had a multivariable hazard ratio for coronary heart disease of 0.95 ( $95 \%$ confidence interval 0.88 to 1.02 ; P for trend $=0.29$ ). After additional adjustment for intake of whole grains (leaving the remaining variance of gluten corresponding to refined grains), the multivariate

\section{WHAT IS ALREADY KNOWN ON THIS TOPIC}

Dietary gluten causes adverse clinical effects in people with celiac disease Avoidance of gluten among people without celiac disease has increased in recent years, partly owing to the belief that gluten can have harmful health effects

\section{WHAT THIS STUDY ADDS}

Among male and female health professionals followed for more than 25 years, quantity of gluten consumption was not associated with coronary heart disease A reduction in dietary gluten may result in the reduced consumption of whole grains, which are associated with lower cardiovascular risk

hazard ratio was 1.00 (0.92 to 1.09; $\mathrm{P}$ for trend=0.77). In contrast, after additional adjustment for intake of refined grains (leaving the variance of gluten intake correlating with whole grain intake), estimated gluten consumption was associated with a lower risk of coronary heart disease (multivariate hazard ratio 0.85 , 0.77 to 0.93 ; $P$ for trend=0.002).

\section{CONCLUSION}

Long term dietary intake of gluten was not associated with risk of coronary heart disease. However, the avoidance of gluten may result in reduced consumption of beneficial whole grains, which may affect cardiovascular risk. The promotion of gluten-free diets among people without celiac disease should not be encouraged.

\section{Introduction}

Gluten, a storage protein in wheat, rye, and barley, triggers inflammation and intestinal damage in people with celiac disease. ${ }^{1}$ People with intestinal or extra-intestinal symptoms triggered by gluten but who do not meet formal criteria for celiac disease may have non-celiac gluten sensitivity, a clinical entity with an as yet uncharacterized biological basis. ${ }^{2}$ Celiac disease, which is present in $0.7 \%$ of the US population, ${ }^{3}$ is associated with an increased risk of coronary heart disease, which is reduced after treatment with a gluten-free diet. ${ }^{4}$

On the basis of evidence that gluten may promote inflammation in the absence of celiac disease or non-celiac gluten sensitivity, ${ }^{5}$ concern has arisen in the medical community and lay public that gluten may increase the risk of obesity, metabolic syndrome, neuropsychiatric symptoms, and cardiovascular risk among healthy people. ${ }^{6-10}$ As a result, diets that limit gluten intake have gained popularity. ${ }^{112}$ In an analysis of the National Health and Nutrition Examination Survey (NHANES), most people adhering to a gluten-free diet did have a diagnosis of celiac disease. ${ }^{3}$ Moreover, in a follow-up analysis of NHANES, adoption of a gluten-free diet by people without celiac disease rose more than threefold from 2009-10 (prevalence 0.52\%) to 201314 (prevalence 1.69\%). ${ }^{13}$

Short of strict gluten avoidance, people may reduce gluten in their diet owing to beliefs that this practice carries general health benefits. ${ }^{14}$ The reasons for gluten reduction likely relate to the perception that gluten carries adverse health effects. One national survey showed a steep rise in interest in this diet in recent years, and by 2013 nearly 30\% of adults in the US reported that they were trying to minimize or avoid gluten. ${ }^{15}$ Concerns exist that a gluten-free or gluten restricted diet may be 
nutritionally suboptimal, ${ }^{16}$ and gluten-free substitute foods cost considerably more than their counterparts that contain gluten. ${ }^{17} 18$ Despite the rising trend in gluten restriction, no long term, prospective studies have assessed the relation of dietary gluten with the risk of chronic conditions such as coronary heart disease in people without celiac disease. Thus, using prospective, validated data on dietary intake collected over 20-30 years, we examined the association of estimated long term intake of gluten with the development of incident coronary heart disease (fatal or non-fatal myocardial infarction).

\section{Methods}

\section{Study population}

The Nurses' Health Study (NHS) is a prospective cohort of 121700 female nurses from 11 US states who were enrolled in 1976. The Health Professionals Follow-up Study (HPFS) is a prospective cohort of 51529 male health professionals from all 50 states who were enrolled in 1986. Participants in NHS and HPFS have been followed via biennial self administered questionnaires on health and lifestyle habits, anthropometrics, environmental exposures, and medical conditions. In 1986, diet in both cohorts was assessed with a validated 136 item semiquantitative food frequency questionnaire. Among the 73666 women in NHS and 49934 men in HPFS who completed a food frequency questionnaire in 1986, we excluded participants if they reported implausible daily energy intake $(<600$ or $>3500 \mathrm{kcal} / \mathrm{d}$ for women and $<800$ or $>4200 \mathrm{kcal} / \mathrm{d}$ for men) or missing gluten data (NHS 48; HPFS 39); a diagnosis of myocardial infarction, angina, or stroke or coronary artery bypass graft surgery (NHS 4015; HPFS 2647); or cancer (NHS 4689; HPFS 1785). Participants were specifically asked about a history of celiac disease in 2014; we excluded from this analysis anyone who reported a previous diagnosis of celiac disease (NHS 200; HPFS 160). After these exclusions, 64714 women and 45303 men were available for analysis. Return of the mailed questionnaire was considered to imply informed consent.

\section{Measurement of exposure and outcome}

In both cohorts, diet was assessed in 1986, 1990, 1994, 1998, 2002, 2006, and 2010. For each food item, participants were asked about the frequency with which they consumed a commonly used portion size for each food over the previous year; available responses ranged from never or less than once a month to six or more times a day. We calculated nutrients by using the Harvard T. H. Chan School of Public Health nutrient database, which was updated every two to four years during the period of food frequency questionnaire distribution. ${ }^{19}$ We used year specific nutrient tables for ingredient level foods. Previous validation studies have shown that the derivation of nutrient values correlates highly with nutrient intake as measured by one week food diaries in women and men. ${ }^{2021}$

For each of these two cohorts, we derived the quantity of gluten consumed. We calculated the quantity of gluten on the basis of the protein content of wheat, rye, and barley based on recipe ingredient lists from product labels provided by manufacturers or cookbooks in the case of home prepared items. Previous studies have used conversion factors of $75 \%$ or $80 \%$ when calculating the proportion of protein content that comprises gluten; we used the more conservative estimate of $75 \% .22-24$ Although gluten's proportion of total protein may be more variable for rye and barley than for wheat, ${ }^{25}$ we used the same conversion factor for all three grains, consistent with previous studies. ${ }^{22} 23$ Although trace amounts of gluten can be present in oats and in condiments (for example, soy sauce), we did not calculate gluten on the basis of these items as the quantity of gluten is much lower than that in cereals and grains and the contribution to total gluten intake would be negligible. ${ }^{26}$

In 1986 the five largest contributors to gluten in both cohorts were dark bread, pasta, cold cereal, white bread, and pizza (supplementary table A). Previous validation studies within these cohorts found that the Pearson correlation coefficients between the number of servings of these items reported on food frequency questionnaires and that reported on seven day dietary records ranged from 0.35 (pasta) to 0.79 (cold cereal) for women and from 0.37 (dark bread) to 0.86 (cold cereal) for men. ${ }^{27} 28$ A separate validation study of this food frequency questionnaire found that this method of measuring vegetable (that is, plant based) protein intake, of which gluten is the major contributor, correlated highly with that measured in seven day dietary records (Spearman correlation coefficient 0.66$).^{29}$

We divided cohort participants into fifths of estimated gluten consumption, according to energy adjusted grams of gluten per day. We obtained energy adjusted values by regression using the residual method, as described previously. ${ }^{30}$ To quantify long term dietary habits, we used cumulative averages through the questionnaires preceding the diagnosis of coronary heart disease, death, or the end of follow-up. ${ }^{31}$ For example, we calculated cumulative average estimated gluten intake in 1994 by averaging the daily consumption of gluten reported in 1986, 1990, and 1994. We treated cumulative average estimated gluten intake as a time varying covariate. For participants with missing dietary data, we used the most recent previous dietary response on record. Because the development of a significant illness may cause a major change in dietary habits, and so as to reduce the possibility of reverse causality, we suspended updating dietary response data for participants who developed diabetes, cardiovascular disease (including stroke, angioplasty, or coronary artery bypass graft surgery), or cancer. For such patients, the cumulative average dietary gluten value before the development of this diagnosis was carried forward until the end of follow-up. ${ }^{32}$

The primary outcome of incident coronary heart disease consisted of a composite outcome of non-fatal myocardial infarction or fatal myocardial infarction. For all participants who recorded such a diagnosis, we requested and reviewed medical records. We classified myocardial infarctions meeting World Health 
Organization criteria, which require typical symptoms plus either diagnostic electrocardiographic findings or elevated cardiac enzyme concentrations, as definite, and we considered myocardial infarctions requiring hospital admission and corroborated by phone interview or letter only as probable. Deaths were identified from state vital records and the National Death Index or reported by participants' next of kin. We classified coronary heart disease deaths by examining autopsy reports, hospital records, or death certificates. Fatal coronary heart disease was confirmed via medical records or autopsy reports or if coronary heart disease was listed as the cause of death on the death certificate and there was previous evidence of coronary heart disease in the medical records. We designated as probable those cases in which coronary heart disease was the underlying cause on the death certificate but no previous knowledge of coronary heart disease was indicated and medical records concerning the death were unavailable. We considered definite and probable myocardial infarction together as our primary outcome, as we have previously found that results were similar when probable cases were excluded. ${ }^{33}$

\section{Statistical analyses}

Patients were followed from 1986 until the development of coronary heart disease, death, or the end of follow-up in 2012 (June 2012 for NHS; January 2012 for HPFS). We tested for the association between cumulative average gluten intake and the development of coronary heart disease, comparing each fifth of gluten intake with the lowest fifth. We used Cox proportional hazards models conditioning on age in months and follow-up cycle to calculate age adjusted and multivariable adjusted hazard ratios and $95 \%$ confidence intervals. We first generated these estimates in each cohort and tested for heterogeneity of the associations by meta-analysis of aggregate data using the $\mathrm{Q}$ statistic. Because we did not observe any significant heterogeneity for the association of gluten with coronary heart disease in the two cohorts ( $\mathrm{P}$ for heterogeneity $>0.10$ ), we then did a pooled analysis combining the participants of NHS and HPFS and estimated the hazard ratios by using Cox modeling stratified by study cohort. We tested the assumption of proportional hazards by testing the interaction term between gluten intake and the period of follow-up and found no violations of this assumption $(\mathrm{P}>0.05)$.

We tested the hypothesis that increasing amounts of energy adjusted dietary gluten is associated with an increased risk of coronary heart disease. Our main model included non-dietary and dietary covariates, constructed a priori. Non-dietary covariates consisted of age, race (white, non-white), body mass index (by fifth), height (in inches), history of diabetes, regular (at least twice weekly) use of aspirin and non-steroidal anti-inflammatory drugs, current use of 3-hydroxy-3-methylglutaryl coenzyme A reductase inhibitors (statins), current use of a multivitamin, smoking history (pack years), parental history of myocardial infarction, history of hypertension, history of hypercholesterolemia, use of physical activity as mea- sured in metabolic equivalents (METs) per week, and (in NHS) menopausal status and menopausal hormone use. Dietary covariates were energy adjusted and consisted of daily consumption of alcohol (grams), trans fats (grams), red meats (servings), processed meats (servings), polyunsaturated fats (grams), fruits (servings), and vegetables (servings).

We did several secondary analyses, constructed a priori. Firstly, because gluten is a component of both refined grains and whole grains, which are each purported to be associated with coronary heart disease, we used multivariable models examining the association between estimated gluten intake and coronary heart disease with additional adjustment for refined grain consumption and whole grain consumption. Secondly, we did stratified analyses by age ( $<65 v \geq 65$ years), body mass index $(<25 v \geq 25)$, physical activity $(<18 v \geq 18$ METhours/week), and smoking status (current $v$ never $v$ past smoking). Thirdly, we separately considered the outcomes of fatal and non-fatal myocardial infarction. Fourthly, we considered the possibility that an association of estimated gluten intake with coronary heart disease may be evident only when extreme levels of intake are considered; we therefore examined participants according to tenths (instead of fifths) of gluten intake. Fifthly, because identification and treatment of risk factors for coronary heart disease may have changed over time, we repeated the primary analysis, restricting the time period first to $1986-97$ and then to $1998-2012$. Sixthly, instead of suspending dietary updates on the diagnosis of cardiovascular disease, diabetes, or cancer (as we did for the primary analysis), we repeated the primary analysis, updating dietary responses regardless of the development of these conditions. Finally, in addition to these a priori analyses, we did post-hoc analyses, including each of the following additional dietary variables in our full model: the Alternate Healthy Eating Index score, percentage protein, percentage total fat, and intake of dairy, saturated fatty acids, monounsaturated fatty acids, sodium, and dietary fiber. We used SAS version 9.4 for all analyses and considered two sided $P$ values of $<0.05$ to be statistically significant.

\section{Patient involvement}

No patients were involved in setting the research question or the outcome measures, nor were they involved in developing plans for recruitment, design, or implementation of the study. No patients were asked to advise on interpretation or writing up of results. Although this specific analysis concerning gluten and coronary heart disease was not conceived in direct collaboration with the research participants, they have been actively engaged in the broad research direction of the cohorts. For example, participants are mailed an annual newsletter that communicates results and highlights notable findings. In response, participants return feedback, including suggestions for future studies. Findings are also disseminated on study websites (www.nurseshealthstudy.org and https://www.hsph.harvard.edu/ hpfs/index.html) 


\section{Results}

Among 64714 women and 45303 men eligible for analysis, the mean daily estimated intake of gluten at baseline was 7.5 (SD 1.4) g among women and 10.0 (2.0) g among men in the highest fifth and $2.6(0.6) \mathrm{g}$ among women and $3.3(0.8) \mathrm{g}$ among men in the lowest fifth. In 2010 the mean daily estimated gluten intake was 7.9 (2.4) g among women and 9.2 (2.8) g among men in the highest fifth and 3.1 (1.2) g among women and 3.7 (1.3) g among men in the lowest fifth. Table 1 shows baseline demographic characteristics according to fifth of gluten intake, and table 2 shows dietary characteristics. Gluten intake correlated inversely with alcohol intake, smoking, total fat intake, and unprocessed red meat intake. Gluten intake correlated positively with whole grain intake (Spearman correlation coefficients NHS 0.37, HPFS 0.42) and refined grain intake (Spearman correlation coefficients NHS 0.66, HPFS 0.65). Gluten did not correlate strongly with sodium intake (Spearman correlation coefficients NHS 0.13, HPFS 0.07).

Over a total of 2273931 person years of follow-up, we documented coronary heart disease in 6529 participants (2431 women and 4098 men). Fatal myocardial infarction developed in 2286 participants (540 women and 1746 men), and non-fatal myocardial infarction developed in 4243 participants (1891 women and 2352 men). Table 3 shows the measurements of association between estimated gluten intake and incident coronary heart disease. Compared with participants in the lowest fifth of gluten intake, who had a coronary heart disease incidence rate of 352 per 100000 person years, those in the highest fifth had a rate of 277 events per 100000 person years, leading to an unadjusted rate difference of 75 (95\% confidence interval 51 to 98 ) fewer cases of coronary heart disease per 100000 person years. With adjustment for age only, participants in the highest fifth of gluten intake had a decreased risk of subsequent coronary heart disease compared with those in the lowest fifth in men (hazard ratio $0.88,95 \%$ confidence interval 0.80 to 0.97$)$ and in the pooled analysis ( $0.87,0.80$ to 0.93). However, after adjustment for race, body mass index, height, diabetes, regular aspirin or non-steroidal anti-inflammatory drug use, statin use, multivitamin use, alcohol, smoking, parental history of coronary heart disease, hypertension, hypercholesterolemia, physical activity, menopausal status, and menopausal hormone use, the association was no longer significant (hazard ratio 0.98, 0.91 to 1.06) in the pooled cohorts.

Addition of other dietary covariates known or purported to be associated with coronary heart disease yielded a similarly null association when we compared participants in the highest fifth of gluten intake with those in the lowest fifth (hazard ratio $0.95,0.88$ to 1.02). Assessment of gluten intake as a continuous variable yielded a multivariate hazard ratio of 0.99 (0.98 to 1.01) for each $1 \mathrm{~g}$ increase in daily intake. In sensitivity analyses, our results were essentially unchanged when we added alternative dietary variables, including Alternate Healthy Eating Index score, percentage protein in the diet, percentage total fat in the diet, and intake of dairy, saturated fatty acids, monounsaturated fatty acids, sodium, or dietary fiber to the model.

\section{Secondary analyses}

As gluten is obtained primarily from whole grains and refined grains, we repeated the primary analysis, adding each of these components to the full model (table 4). When further adjusting for refined grains (with the

\begin{tabular}{|c|c|c|c|c|c|c|}
\hline \multirow[b]{2}{*}{ Characteristics } & \multicolumn{3}{|c|}{ Nurses' Health Study (1986) } & \multicolumn{3}{|c|}{ Health Professionals Follow-up Study (1986) } \\
\hline & $\begin{array}{l}1 \text { (lowest) } \\
(n=12449)\end{array}$ & $3(n=12909)$ & $\begin{array}{l}5 \text { (highest) } \\
(\mathrm{n}=13236)\end{array}$ & $\begin{array}{l}1 \text { (lowest) } \\
(\mathrm{n}=9120)\end{array}$ & $3(n=9057)$ & $\begin{array}{l}5 \text { (highest) } \\
(\mathrm{n}=8993)\end{array}$ \\
\hline Median (range) gluten intake, $\mathrm{g} / \mathrm{d}$ & $2.8(0-3.4)$ & $4.7(4.3-5.1)$ & $7.1(6.2-26.7)$ & $3.5(0-4.3)$ & $6.0(5.5-6.6)$ & $9.4(8.1-38.4)$ \\
\hline Mean (SD) age, years & $53.0(6.9)$ & $51.9(7.2)$ & $52.3(7.3)$ & $54.6(9.6)$ & $53.2(9.6)$ & $53.2(9.7)$ \\
\hline White race & $11915(96)$ & $12692(98)$ & $13073(99)$ & $8031(88)$ & $8277(91)$ & $8310(92)$ \\
\hline Median (IQR) physical activity, METs ${ }^{*}$ & $7.7(2.5-20.2)$ & $7.7(2.9-18.7)$ & $7.7(2.5-17.9)$ & $10.4(3.1-26.3)$ & $11.8(4.1-27.6)$ & $14.3(4.8-31.3)$ \\
\hline Mean (SD) body mass index ${ }^{\dagger}$ & $25.3(4.8)$ & $25.3(4.8)$ & $25.0(4.7)$ & $25.9(3.6)$ & $25.6(3.3)$ & $24.9(3.1)$ \\
\hline Mean (SD) height, inches ${ }^{\dagger}$ & $64.5(2.4)$ & $64.6(2.4)$ & $64.5(2.4)$ & $70.1(3.5)$ & $70.1(3.2)$ & $70.0(3.2)$ \\
\hline Mean (SD) alcohol, g/d $\mathrm{d}^{+}$ & $8.4(13.6)$ & $6.0(10.0)$ & $4.3(8.0)$ & $13.2(17.9)$ & $11.9(15.6)$ & $8.5(11.9)$ \\
\hline Past or current smoking & $7480(60)$ & $7096(55)$ & $6957(53)$ & $5146(58)$ & $4606(53)$ & $4129(48)$ \\
\hline Mean (SD) pack years of smoking (among past or current smokers) ${ }^{\dagger}$ & $24.7(19.2)$ & $22.2(18.5)$ & $21.1(18.2)$ & $26.8(19.3)$ & $24.3(18.6)$ & $22.7(18.3)$ \\
\hline History of diabetes, & $517(4.0)$ & $384(3.0)$ & $405(3.0)$ & $294(3.0)$ & $227(2.6)$ & $239(2.7)$ \\
\hline History of hypertension & $3398(27)$ & $2965(23)$ & $2989(23)$ & $2226(24)$ & $1864(21)$ & $1755(20)$ \\
\hline History of hypercholesterolemia & $1446(11)$ & $1405(11)$ & 1654 (13) & $973(11)$ & $986(11)$ & $1155(13)$ \\
\hline Parental history of myocardial infarction & $4728(38)$ & 4958 (39) & $5091(39)$ & $2918(32)$ & $2936(33)$ & $3015(35)$ \\
\hline Regular aspirin use & $4183(37)$ & $4334(36)$ & $4360(36)$ & $2565(28)$ & $2503(28)$ & $2515(28)$ \\
\hline Regular NSAID use & $3862(36)$ & $4283(37)$ & $4219(36)$ & $541(6)$ & $464(5)$ & $438(5)$ \\
\hline Regular statin use & $241(2)$ & $234(2)$ & $278(2)$ & $48(0.6)$ & $52(0.5)$ & $64(0.7)$ \\
\hline Current multivitamin use & $5278(43)$ & $5463(43)$ & $5651(43)$ & $3776(42)$ & $3782(43)$ & $3905(44)$ \\
\hline Menopausal status & $8812(68)$ & $8290(66)$ & 8609 (65) & - & - & - \\
\hline Current menopausal hormone use & $2143(17)$ & $2154(17)$ & $2301(18)$ & - & - & - \\
\hline
\end{tabular}




\begin{tabular}{|c|c|c|c|c|c|c|}
\hline \multirow[b]{2}{*}{ Characteristics } & \multicolumn{3}{|c|}{ Nurses' Health Study (1986) } & \multicolumn{3}{|c|}{ Health Professionals Follow-up Study (1986) } \\
\hline & $\begin{array}{l}1 \text { (lowest) } \\
(n=12449)\end{array}$ & $3(n=12909)$ & $\begin{array}{l}5 \text { (highest) } \\
(\mathrm{n}=13236)\end{array}$ & $\begin{array}{l}1 \text { (lowest) } \\
(n=9120)\end{array}$ & $3(n=9057)$ & $\begin{array}{l}5 \text { (highest) } \\
(\mathrm{n}=8993)\end{array}$ \\
\hline Median (range) gluten intake, g/d & $2.8(0-3.4)$ & $4.7(4.3-5.1)$ & $7.1(6.2-26.7)$ & $3.5(0-4.3)$ & $6.0(5.5-6.6)$ & $9.4(8.1-38.4)$ \\
\hline Total energy, kcal & $1738(554)$ & $1815(525)$ & $1683(492)$ & $1966(654)$ & $2045(619)$ & $1904(583)$ \\
\hline Energy adjusted gluten intake, $\mathrm{g} / \mathrm{d}$ & $2.6(0.6)$ & $4.7(0.2)$ & $7.5(1.4)$ & $3.3(0.8)$ & $6.0(0.3)$ & $10.0(2.0)$ \\
\hline Median (IQR) whole grains, g/d & $5.9(2.6-11.2)$ & $10.3(5.2-17.1)$ & $20.7(10.6-32.9)$ & $9.4(4.1-17.9)$ & $16.1(8.6-25.9)$ & $32.7(19.0-48.3)$ \\
\hline Refined grains, $\mathrm{g} / \mathrm{d}$ & $31.7(12.5)$ & $47.0(10.8)$ & $61.4(18.3)$ & $39.0(18.8)$ & $57.6(15.6)$ & $77.1(24.6)$ \\
\hline Cereal fiber, g/d & $2.4(1.4)$ & $4.1(1.9)$ & $7.2(4.4)$ & $3.2(2.0)$ & $5.4(2.5)$ & $9.7(5.6)$ \\
\hline Median (IQR) bran, g/d & $0.8(0.3-1.9)$ & $1.7(0.7-3.5)$ & $3.9(1.6-7.8)$ & $1.2(0.4-2.9)$ & $2.6(1.1-5.7)$ & $6.3(2.9-11.6)$ \\
\hline Polyunsaturated fat, $\mathrm{g} / \mathrm{d}$ & $10.8(3.4)$ & $11.0(2.7)$ & $10.9(2.7)$ & $13.3(4.1)$ & $13.3(3.3)$ & $12.9(3.3)$ \\
\hline Trans fat, $\mathrm{g} / \mathrm{d}$ & $2.2(0.8)$ & $2.5(0.9)$ & $2.6(1.0)$ & $2.6(1.0)$ & $2.9(1.1)$ & $2.8(1.2)$ \\
\hline Sodium, mg/d & 2759 (1144) & 2849 (999) & $2836(967)$ & $3229(1325)$ & $3276(1086)$ & $3261(1059)$ \\
\hline Glycemic load & $90.4(22.9)$ & $97.7(16.2)$ & $108.7(16.2)$ & $112.2(29.7)$ & $122.6(22.5)$ & $140.2(22.4)$ \\
\hline Glycemic index & $50.5(4.6)$ & $52.1(3.2)$ & $53.7(3.0)$ & $51.7(4.5)$ & $53.1(3.2)$ & $54.7(3.0)$ \\
\hline Median (IQR) processed meat, servings/week & $1.4(0.5-2.5)$ & $1.5(0.9-3.0)$ & $1.4(0.5-2.5)$ & $1.5(0.9-4.0)$ & $1.9(0.9-3.9)$ & $1.0(0.5-2.5)$ \\
\hline Carbohydrate, \% of energy (median) & 45.6 & 47.7 & 51.2 & 43.2 & 46.0 & 51.0 \\
\hline Protein, \% of energy (median) & 19.1 & 18.4 & 17.9 & 19.0 & 18.3 & 18.0 \\
\hline Total fat, \% of energy (median) & 33.3 & 33.2 & 31.2 & 34.0 & 32.7 & 29.4 \\
\hline Unprocessed red meat, servings/week & $4.4(3.2)$ & $4.2(2.6)$ & $3.3(2.2)$ & $5.0(3.8)$ & $4.5(3.1)$ & $3.1(2.6)$ \\
\hline Vegetables, servings/d & $4.3(2.6)$ & $4.0(2.1)$ & $3.5(1.9)$ & $3.6(2.4)$ & $3.4(1.9)$ & $3.2(1.9)$ \\
\hline Fruits, servings/d & $1.9(1.5)$ & $1.8(1.2)$ & $1.5(1.0)$ & $1.7(1.6)$ & $1.6(1.2)$ & $1.6(1.2)$ \\
\hline Alternate Healthy Eating Index score ${ }^{\star}$ & $51.9(11.9)$ & $51.2(10.8)$ & $52.4(11.0)$ & $52.0(11.9)$ & $52.1(11.2)$ & $55.0(11.5)$ \\
\hline
\end{tabular}

\section{IQR=interquartile range}

*Score of diet quality that incorporates vegetables, fruits, whole grains, sugar sweetened drinks and juices, nuts and legumes, red and/or processed meat, and alcohol; score ranges from 0

(lowest quality) to 110 (highest quality).

Table 3 | Gluten and risk of coronary heart disease (fatal and non-fatal myocardial infarctions)

\begin{tabular}{|c|c|c|c|c|c|c|}
\hline & \multicolumn{5}{|c|}{ Fifth of energy adjusted gluten intake } & \multirow[b]{2}{*}{ P for trend } \\
\hline & 1 (lowest) & 2 & 3 & 4 & 5 (highest) & \\
\hline \multicolumn{7}{|l|}{ Nurses' Health Study } \\
\hline Mean; median (range) gluten intake, g/d & $2.6 ; 2.8(0-3.4)$ & $3.8 ; 3.8(3.4-4.3)$ & $4.7 ; 4.7(4.3-5.1)$ & $5.6 ; 5.6(5.1-6.2)$ & $7.5 ; 7.1(6.2-26.7)$ & - \\
\hline No of events & 492 & 470 & 494 & 471 & 504 & - \\
\hline Person years & 246539 & 280655 & 290265 & 296789 & 293279 & - \\
\hline Incidence per 100000 person years & 200 & 167 & 170 & 159 & 172 & - \\
\hline Age adjusted HR (95\% Cl) & 1.0 (reference) & 0.88 (0.77 to 1.00$)$ & 0.90 (0.80 to 1.02$)$ & $0.84(0.74$ to 0.96$)$ & 0.89 (0.79 to 1.01$)$ & 0.08 \\
\hline Multivariable adjusted HR $(95 \% \mathrm{CI})^{*}$ & 1.0 (reference) & 0.97 (0.86 to 1.10$)$ & $1.02(0.90$ to 1.16$)$ & $0.97(0.85$ to 1.10$)$ & $1.00(0.88$ to 1.14$)$ & 0.98 \\
\hline Full model HR $(95 \% \mathrm{Cl})^{\dagger}$ & 1.0 (reference) & 0.96 (0.85 to 1.10$)$ & $1.02(0.90$ to 1.16$)$ & 0.96 (0.84 to 1.09$)$ & 1.01 (0.89 to 1.15$)$ & 0.92 \\
\hline \multicolumn{7}{|l|}{ Health Professionals Follow-up Study } \\
\hline Mean; median (range) gluten intake, g/d & $3.3 ; 3.5(0-4.3)$ & $4.9 ; 4.9(4.3-5.5)$ & $6.0 ; 6.0(5.5-6.6)$ & $7.3 ; 7.3(6.6-8.1)$ & $10.0 ; 9.4(8.1-38.4)$ & - \\
\hline No of events & 930 & 768 & 849 & 756 & 795 & - \\
\hline Person years & 157910 & 172630 & 179552 & 180670 & 175641 & - \\
\hline Incidence per 100000 person years & 589 & 445 & 473 & 418 & 453 & - \\
\hline Age adjusted HR $(95 \% \mathrm{Cl})$ & 1.0 (reference) & 0.81 (0.74 to 0.89$)$ & 0.89 (0.81 to 0.98$)$ & 0.80 (0.73 to 0.89$)$ & $0.88(0.80$ to 0.97$)$ & 0.02 \\
\hline Multivariable adjusted HR $(95 \% \mathrm{Cl})^{*}$ & 1.0 (reference) & 0.86 (0.78 to 0.95$)$ & 0.96 (0.88 to 1.06$)$ & 0.89 (0.80 to 0.98$)$ & 0.98 (0.89 to 1.07$)$ & 0.78 \\
\hline Full model HR $(95 \% \mathrm{Cl})^{+}$ & 1.0 (reference) & 0.86 (0.78 to 0.94$)$ & 0.95 (0.87 to 1.05$)$ & $0.87(0.79$ to 0.96$)$ & 0.96 (0.87 to 1.05$)$ & 0.49 \\
\hline \multicolumn{7}{|l|}{ Pooled } \\
\hline No of events & 1422 & 1238 & 1343 & 1227 & 1299 & - \\
\hline Person years & 404450 & 453285 & 469817 & 477459 & 468920 & - \\
\hline Incidence per 100000 person years & 352 & 273 & 286 & 257 & 277 & - \\
\hline Age adjusted HR (95\% Cl) & 1.0 (reference) & 0.83 (0.77 to 0.90$)$ & 0.89 (0.83 to 0.96$)$ & 0.81 (0.75 to 0.87$)$ & 0.87 (0.80 to 0.93$)$ & 0.001 \\
\hline Multivariable adjusted HR $(95 \% \mathrm{CI})^{\star}$ & 1.0 (reference) & 0.90 (0.83 to 0.97$)$ & 0.98 (0.91 to 1.06$)$ & 0.91 (0.84 to 0.98$)$ & 0.98 (0.91 to 1.06$)$ & 0.81 \\
\hline Full model HR $(95 \% \mathrm{Cl})^{\dagger}$ & 1.0 (reference) & $0.88(0.82$ to 0.95$)$ & 0.96 (0.89 to 1.04$)$ & 0.88 (0.82 to 0.95$)$ & 0.95 (0.88 to 1.02$)$ & 0.29 \\
\hline
\end{tabular}

HR=hazard ratio.

${ }^{*}$ Additionally adjusted for race, body mass index, height, history of diabetes, regular non-steroidal anti-inflammatory drug use, current use of multivitamin, alcohol intake (g/d), smoking (pack years), aspirin use, statin use, parental history of myocardial infarction, history of hypertension, history of hypercholesterolemia, physical activity (MET, h/wk), menopausal status (Nurses' Health Study only), and menopausal hormone use (Nurses' Health Study only).

tAbove model additionally adjusted for trans fat, red meat, processed meat, polyunsaturated fats, fruits, and vegetables. 


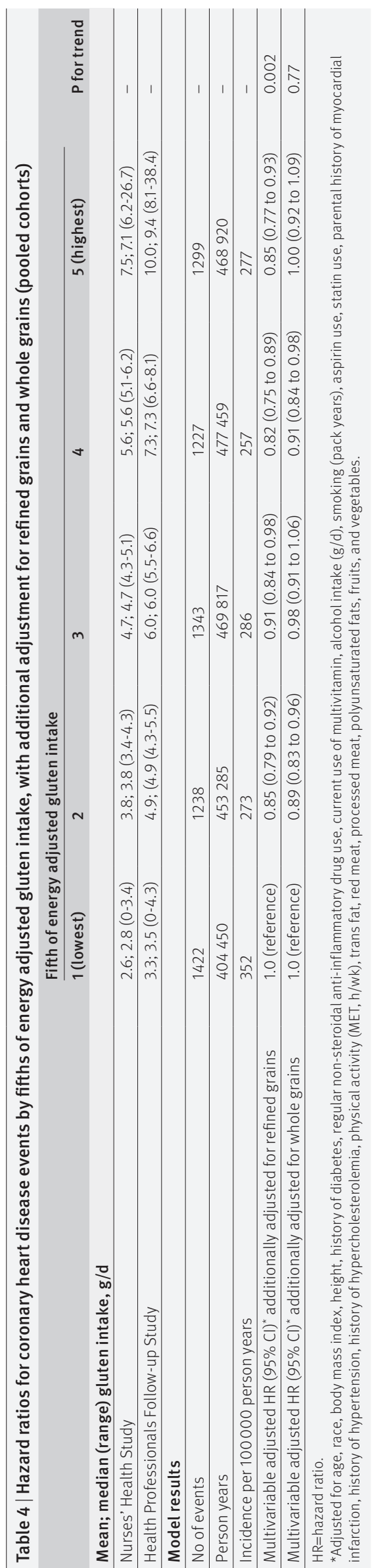

remaining variance of gluten intake correlating with whole grain intake), we found an inverse relation between estimated gluten intake and coronary heart disease; participants in the highest fifth of gluten intake had a lower coronary heart disease risk (hazard ratio $0.85,0.77$ to 0.93$)$. When we instead adjusted for whole grains (leaving the variance of gluten intake correlating with refined grain intake), we found no association between gluten intake and incident coronary heart disease; participants in the highest fifth of gluten intake had a risk of coronary heart disease that was not different from those in the lowest group (hazard ratio 1.00, 0.92 to 1.09 ).

Table 5 shows results according to subgroups defined by age, body mass index, physical activity, and smoking status. The association between estimated gluten intake and coronary heart disease remained null across all of these strata with the exception of smoking status. Among current smokers, the highest fifth of gluten intake was associated with increased risk of coronary heart disease (hazard ratio 1.34, 1.09 to 1.66; P for trend=0.02). However, when we additionally adjusted for refined grains (leaving the variance of gluten intake correlating with whole grain intake), the association between gluten intake and coronary heart disease was no longer significant (hazard ratio for highest fifth 1.25, 0.95 to 1.64; $P$ for trend=0.21).

We found no significant association between estimated gluten intake and either fatal myocardial infarction or non-fatal myocardial infarction when considered as separate outcomes (see supplementary table B). We did not observe any significant associations between tenth categories of gluten intake with risk of coronary heart disease (see supplementary table C). Nor did we find a significant association between gluten intake and coronary heart disease when separately considering the time strata 1986-97 and 1998-2012 or when updating dietary responses regardless of the development of the comorbid conditions of cardiovascular disease, diabetes, or cancer (see supplementary tables D and E).

\section{Discussion}

In two prospective cohorts with updated dietary information over 20 years of follow-up, we found no significant association between estimated gluten intake and the risk of subsequent overall coronary heart disease, non-fatal myocardial infarction, and fatal myocardial infarction. The lack of association was consistent in both men and women, as well among other subgroups defined by cardiovascular risk factors.

\section{Comparison with other studies}

Dietary gluten has been the subject of increased attention and concern in recent years. Much of the data on gluten and coronary heart disease are limited to people with celiac disease, in whom gluten elicits an inflammatory response characterized by small intestinal villous atrophy and the development of antibodies to tissue transglutaminase, a ubiquitous enzyme that is present on vascular endothelial cells. ${ }^{345}$ Patients with celiac disease may have an increased risk of myocardial 
Table 5 | Hazard ratios for coronary heart disease events (fatal and non-fatal myocardial infarctions) by fifths of energy adjusted gluten intake, stratified by age, body mass index, physical activity, and smoking

Fifth of energy adjusted gluten intake

1 (lowest) $\quad 2$

Mean; median (range) gluten intake, g/d

Nurses' Health Study

$2.6 ; 2.8(0-3.4) \quad 3.8 ; 3.8(3.4-4.3)$

3

4 (highest)

P for P for

$4.7 ; 4.7(4.3-5.1)$

$5.6 ; 5.6(5.1-6.2)$

$7.5 ; 7.1(6.2-26.7)$

Health Professionals Follow-up Study

$3.3 ; 3.5(0-4.3) \quad 4.9 ;(4.9(4.3-5.5)$

$6.0 ; 6.0(5.5-6.6) \quad 7.3 ; 7.3(6.6-8.1)$

$10.0 \cdot 9.4(8.1-38.4)$

Age

$<65$ years:

\begin{tabular}{|c|c|c|c|c|c|c|}
\hline No of events & 469 & 423 & 460 & 404 & 438 & - \\
\hline Person years & 231320 & 269283 & 282702 & 288484 & 282443 & - \\
\hline Incidence per 100000 person years & 203 & 157 & 163 & 140 & 155 & - \\
\hline Multivariable adjusted HR $(95 \% \mathrm{CI})^{*}$ & 1.0 (reference) & 0.85 (0.75 to 0.97$)$ & 0.89 (0.78 to 1.01) & 0.78 (0.69 to 0.90$)$ & 0.88 (0.77 to 1.00$)$ & 0.07 \\
\hline \multicolumn{7}{|l|}{$\geq 65$ years: } \\
\hline No of events & 953 & 815 & 883 & 823 & 861 & - \\
\hline Person years & 173129 & 183989 & 187115 & 188976 & 186478 & - \\
\hline Incidence per 100000 person years & 550 & 443 & 472 & 436 & 462 & - \\
\hline Multivariable adjusted HR $(95 \% \mathrm{CI})^{\star}$ & 1.0 (reference) & $0.90(0.82$ to 0.99$)$ & 1.00 (0.91 to 1.09) & 0.94 (0.85 to 1.03$)$ & $0.98(0.90$ to 1.08$)$ & 0.97 \\
\hline
\end{tabular}

\section{Body mass index}

$<25$ :

\begin{tabular}{|c|c|c|c|c|c|c|c|}
\hline No of events & 516 & 463 & 540 & 521 & 570 & - & \multirow{9}{*}{0.36} \\
\hline Person years & 188630 & 212146 & 225115 & 241864 & 258489 & - & \\
\hline Incidence per 100000 person years & 274 & 218 & 240 & 215 & 221 & - & \\
\hline Multivariable adjusted HR $(95 \% \mathrm{CI})^{\star}$ & 1.0 (reference) & 0.90 (0.79 to 1.02$)$ & $1.00(0.89$ to 1.14$)$ & 0.90 (0.80 to 1.02$)$ & 0.91 (0.81 to 1.03$)$ & 0.16 & \\
\hline \multicolumn{7}{|l|}{$\geq 25$} & \\
\hline No of events & 899 & 768 & 798 & 702 & 727 & - & \\
\hline Person years & 214254 & 239945 & 243559 & 234408 & 209234 & - & \\
\hline Incidence per 100000 person years & 420 & 320 & 328 & 299 & 347 & - & \\
\hline Multivariable adjusted HR $(95 \% \mathrm{CI})^{\star}$ & 1.0 (reference) & $0.87(0.79$ to 0.96$)$ & $0.94(0.85$ to 1.04$)$ & 0.88 (0.80 to 0.98$)$ & $1.00(0.90$ to 1.10$)$ & 0.87 & \\
\hline \multicolumn{8}{|l|}{ Physical activity } \\
\hline \multicolumn{7}{|l|}{ <18 METS/week: } & \multirow{10}{*}{0.77} \\
\hline No of events & 858 & 743 & 730 & 724 & 739 & - & \\
\hline Person years & 235824 & 260756 & 268065 & 272513 & 266980 & - & \\
\hline Incidence per 100000 person years & 364 & 285 & 272 & 266 & 277 & - & \\
\hline Multivariable adjusted HR $(95 \% \mathrm{CI})^{\star}$ & 1.0 (reference) & $0.91(0.82$ to 1.00$)$ & 0.91 (0.82 to 1.01) & $0.91(0.82$ to 1.01$)$ & 0.92 (0.83 to 1.02) & 0.16 & \\
\hline \multicolumn{7}{|l|}{$\geq 18$ METS/week: } & \\
\hline No of events & 563 & 493 & 610 & 503 & 558 & - & \\
\hline Person years & 167954 & 192090 & 201348 & 204611 & 201627 & - & \\
\hline Incidence per 100000 person years & 335 & 257 & 303 & 246 & 277 & - & \\
\hline Multivariable adjusted HR $(95 \% \mathrm{Cl})^{\star}$ & 1.0 (reference) & $0.85(0.75$ to 0.96$)$ & $1.03(0.92$ to 1.16$)$ & $0.86(0.76$ to 0.97$)$ & 1.00 (0.89 to 1.13$)$ & 0.79 & \\
\hline
\end{tabular}

\section{Smoking history}

Never smokers:

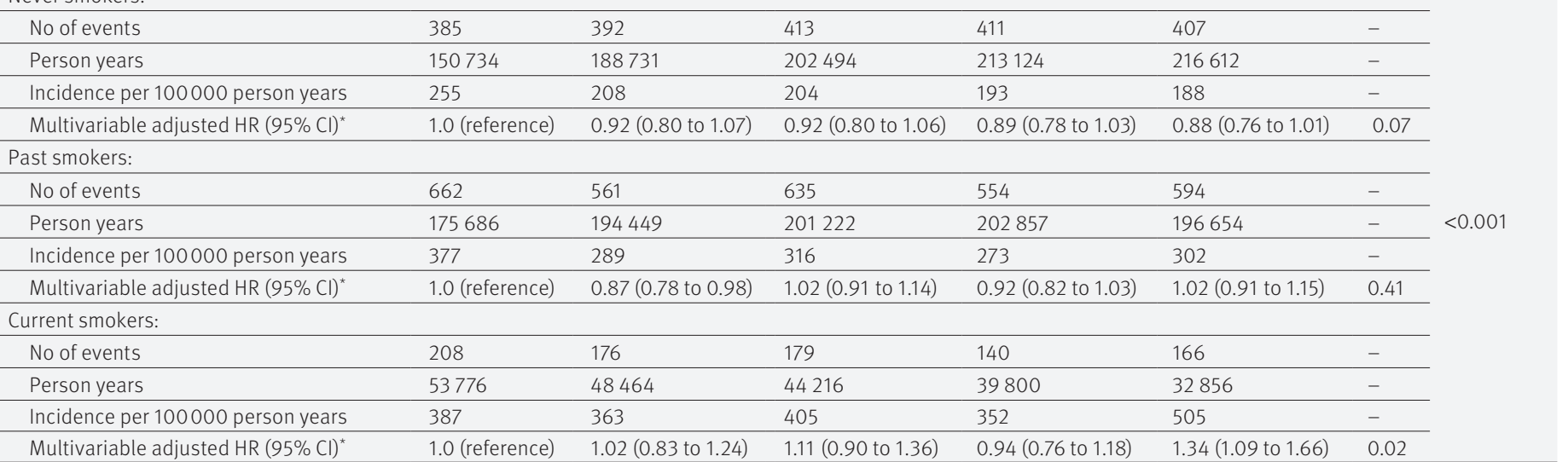

*Adjusted for age, race, body mass index, height, history of diabetes, regular non-steroidal anti-inflammatory drug use, current use of multivitamin, alcohol intake (g/d), smoking (pack years), aspirin use, statin use, parental history of myocardial infarction, history of hypertension, history of hypercholesterolemia, physical activity (MET, h/wk), menopausal status and menopausal

hormone use (Nurses' Health Study only), trans fat, red meat, processed meat, polyunsaturated fats, fruits, and vegetables. 
infarction and death from cardiovascular disease that is reduced after diagnosis of celiac disease, possibly owing to the beneficial effect of a gluten-free diet, ${ }^{436}$ although this association is controversial. ${ }^{37}$ Patients with celiac disease who develop a myocardial infarction are less likely to have classic cardiac risk factors, such as smoking and dyslipidemia, leading to the hypothesis that the pro-inflammatory effect of gluten exerts an independent cardiac risk. ${ }^{38}$

The popularity of a low gluten or gluten-free diet in the general population has markedly increased in recent years. ${ }^{13}$ Despite the limited evidence that gluten plays a role in cardiovascular health, this increasing adoption of a gluten-free diet by people without celiac disease has occurred in conjunction with speculation that gluten may have a deleterious role in health outcomes even in the absence of gluten sensitivity. The rationale for this concern includes the observation that foods containing gluten often have a high glycemic index, which has been linked to cardiovascular risk. ${ }^{11}$ Studies in mice have shown pro-inflammatory effects of gluten administration and a protective association of gluten restriction with the development of diabetes. ${ }^{39} 40$ In one cross sectional study in young adults, gluten intake was correlated with higher plasma concentrations of $\alpha 2$-macroglobulin, an acute phase reactant that is associated with inflammation. ${ }^{5}$ Gluten has been found to cause gastrointestinal symptoms in patients without celiac disease, ${ }^{41}$ although the mechanism for this remains uncertain. ${ }^{42} 43$

We found that estimated gluten intake correlated moderately with whole grain and refined grain intake, as expected given the prominence of wheat among dietary grains; gluten also correlated with glycemic index. We noted a significant inverse relation between estimated gluten intake and coronary heart disease when we adjusted for refined grain intake. Although the absolute risk difference was modest (75 coronary heart disease events per 100000 person years when we compared the highest fifth of gluten intake with the lowest fifth in the pooled analysis), this lower risk likely reflects the fact that adjustment for refined grains leaves the remainder of the variance of gluten intake correlated with whole grain intake. Whole grain intake has been found to be inversely associated with coronary heart disease risk and cardiovascular mortality. ${ }^{4445}$ These findings underscore the potential that people who severely restrict gluten intake may also significantly limit their intake of whole grains, which may actually be associated with adverse cardiovascular outcomes.

\section{Strengths and limitations of study}

Strengths of our study include its large sample size, long term follow-up, prospective and repeated assessments of diet with validated questionnaires, and validated outcome measurement. Our study also has several limitations. Unmeasured or residual negative confounding is a possibility, although our main model included multiple dietary and non-dietary covariates. We did not specifically ask about the intake of gluten-free substitute foods, and participants were not asked about whether they specifically adhered to a gluten-free diet. Although we excluded participants who reported a diagnosis of celiac disease, we could not identify which people without celiac disease nonetheless maintained a very low gluten or gluten-free diet. Nevertheless, the observation period (1986-2012) largely preceded the widespread interest in gluten as a health concern that has arisen more recently in the US. ${ }^{11-13}$ We likewise were unable to determine whether gluten was present in trace amounts in certain foods, such as soy sauce or oats that were not harvested on separate fields; therefore, potential exists for misclassification at the low end of gluten intake. Although trace amounts of gluten (such as 50 mg daily) can induce symptoms and inflammation in patients with celiac disease, ${ }^{46}$ measurement of such gluten exposure would have a small effect on gluten quantity even in the lowest fifth of baseline daily gluten intake in our cohorts (2.6 g daily in women and $3.3 \mathrm{~g}$ in men). Therefore, although we were unable to determine the association of a strict gluten-free diet with coronary heart disease, we did not observe any association of very low estimated gluten intake with coronary heart disease, as might be realistically expected among people who maintain a gluten-free diet in usual practice.

Our measurement of gluten intake was based on the assumption that gluten comprised $75 \%$ of the protein content of wheat, rye, and barley, following the convention of a single conversion factor for all three grains. ${ }^{22} 23$ Although this may overestimate the amount of gluten intake for rye and barley, it is unlikely to bias our results given the overall low intake of rye and barley in these cohorts. Although gluten has not been specifically quantified in validation studies of food frequency questionnaires, this instrument has shown good validity with regard to reasonable correlation with seven day dietary recall of foods containing gluten (supplementary table A) and intake of vegetable protein, to which gluten is a significant contributor. ${ }^{29}$ In addition, participants with undiagnosed celiac disease were not uniformly identified in these cohorts. However, according to population based estimates, such people would account for less than $1 \%$ of the cohort. ${ }^{3}$ Moreover, inclusion of these participants would be expected to bias the results toward an association of gluten with coronary heart disease, which was not observed.

In this analysis, we did not examine change in body mass index in relation to gluten ingestion. However, body mass index is unlikely to mediate an association between gluten and coronary heart disease, as the risk estimate did not change from positive toward a null association when we added body mass index to the model. Finally, in secondary subgroup analyses, we observed a higher risk of coronary heart disease among participants in the highest fifth of gluten intake among current smokers. However, these associations were no longer significant once we adjusted the models for refined grain consumption. Given the relatively small number of cases of coronary heart disease among current smokers in the highest fifth of gluten intake and the 
lack of a clear mechanistic basis for this heterogeneity, these results should be viewed in the context of multiple testing. When we applied the Bonferroni correction to smoking categories (which contained three strata), our finding regarding gluten intake and coronary heart disease among current smokers was no longer statistically significant.

\section{Conclusion and public health implications}

In these two large, prospective cohorts, the consumption of foods containing gluten was not significantly associated with risk of coronary heart disease. Although people with and without celiac disease may avoid gluten owing to a symptomatic response to this dietary protein, these findings do not support the promotion of a gluten restricted diet with a goal of reducing coronary heart disease risk. In addition, the avoidance of dietary gluten may result in a low intake of whole grains, which are associated with cardiovascular benefits. The promotion of gluten-free diets for the purpose of coronary heart disease prevention among asymptomatic people without celiac disease should not be recommended.

We thank the participants and staff of the Nurses' Health Study and Health Professionals Follow-up Study for their contributions and Elaine Coughlin-Gifford and Mingyang Song for statistical programming.

Contributors: All authors were involved in study concept and design. $\mathrm{BL}, \mathrm{YC}, \mathrm{GZ}, \mathrm{LS}, \mathrm{LWD}$, and ATC were involved in acquisition of data. BL, YC, GZ, All authors analyzed and interpreted the data. BL, YC, GZ ATC did the statistical analysis. BL and ATC drafted the manuscript. All authors critically revised the manuscript for important intellectual content. All authors approved the final manuscript submitted and the authorship list. ATC is the guarantor.

Funding: BL is supported by the American Gastroenterological Association's foundation research scholar award. ATC is supported by the Massachusetts General Hospital research scholars award and National Institutes of Health (NIH) grants K24 DK098311 and UM1 CA186107. WCW is supported by NIH grant UM1 CA167552, EBR by grant R01 HL035464, QS by grant R01 HL035464, and FBH by grants R01 HL060712 and R01 HL034594.

Competing interests: All authors have completed the ICMJE uniform disclosure form at www.icmje.org/coi_disclosure.pdf (available on request from the corresponding author) and declare: no support from any organization for the submitted work apart from the grant support noted above; no financial relationships with any organizations that might have an interest in the submitted work in the previous three years; no other relationships or activities that could appear to have influenced the submitted work.

Ethical approval: The Institutional Review Boards of Brigham and Women's Hospital and the Harvard T. H. Chan School of Public Health approved this study. Return of the mailed questionnaire was considered to imply informed consent. Protocol number: 1999-P-011114/154.

Transparency declaration: The lead author (the manuscript's guarantor) affirms that the manuscript is an honest, accurate, and transparent account of the study being reported; that no important aspects of the study have been omitted; and that any discrepancies from the study as planned (and, if relevant, registered) have been explained.

Data sharing: Data, the statistical code, questionnaires, and technical processes are available from the corresponding author at achan@ mgh.harvard.edu.

This is an Open Access article distributed in accordance with the Creative Commons Attribution Non Commercial (CC BY-NC 4.0) license, which permits others to distribute, remix, adapt, build upon this work non-commercially, and license their derivative works on different terms, provided the original work is properly cited and the use is non-commercial. See: http://creativecommons.org/licenses/ by-nc/4.0/.

1 Lebwohl B, Ludvigsson JF, Green PH. Celiac disease and non-celiac gluten sensitivity. BMJ 2015;351:h4347. doi:10.1136/bmi.h4347.
2 Fasano A, Sapone A, Zevallos V, Schuppan D. Nonceliac gluten sensitivity. Gastroenterology 2015;148:1195-204. doi:10.1053/j. gastro.2014.12.049.

3 Rubio-Tapia A, Ludvigsson JF, Brantner TL, Murray JA, Everhart JE. The prevalence of celiac disease in the United States. Am J Gastroenterol 2012;107:1538-44, quiz 1537, 1545. doi:10.1038/ajg.2012.219.

4 Ludvigsson JF, James S, Askling J, Stenestrand U, Ingelsson E. Nationwide cohort study of risk of ischemic heart disease in patients with celiac disease. Circulation 2011;123:483-90. doi:10.1161/ CIRCULATIONAHA.110.96562

5 Jamnik J, García-Bailo B, Borchers CH, El-Sohemy A. Gluten Intake is Positively Associated with Plasma $\alpha 2$-Macroglobulin in Young Adults J Nutr 2015;145:1256-62. doi:10.3945/in.115.212829.

6 Soares FL, de Oliveira Matoso R, Teixeira LG, et al. Gluten-free diet reduces adiposity, inflammation and insulin resistance associated with the induction of PPAR-alpha and PPAR-gamma expression. J Nutr Biochem 2013:24:1105-11. doi:10.1016/i.jnutbio.2012.08.009.

7 Schiltz B, Minich DM, Lerman RH, Lamb JJ, Tripp ML, Bland JS. A science-based, clinically tested dietary approach for the metabolic syndrome. Metab Syndr Relat Disord 2009;7:187-92. doi:10.1089/ met.2008.0051.

8 Eaton WW, Chen LY, Dohan FC Jr,, Kelly DL, Cascella N. Improvement in psychotic symptoms after a gluten-free diet in a boy with complex autoimmune illness. Am J Psychiatry 2015;172:219-21. doi:10.1176/ appi.ajp.2014.14040550

9 Peters SL, Biesiekierski JR, Yelland GW, Muir JG, Gibson PR. Randomised clinical trial: gluten may cause depression in subjects with non-coeliac gluten sensitivity - an exploratory clinical study. Aliment Pharmacol Ther 2014;39:1104-12. doi:10.1111/apt.12730.

10 Elkan AC, Sjöberg B, Kolsrud B, Ringertz B, Hafström I, Frostegård J. Gluten-free vegan diet induces decreased LDL and oxidized LDL levels and raised atheroprotective natural antibodies against phosphorylcholine in patients with rheumatoid arthritis: randomized study. Arthritis Res Ther 2008;10:R34. doi:10.1186/ ar2388.

11 Davis W. Wheat belly: lose the wheat, lose the weight, and find your path back to health. Rodale, 2011.

12 Perlmutter D, Loberg K. Grain brain: the surprising truth about wheat, carbs, and sugar-your brain's silent killers. Little, Brown \& Company, 2013

$13 \mathrm{Kim} \mathrm{HS}$, Patel KG, Orosz E, et al. Time trends in the prevalence of celiac disease and gluten-free diet in the us population: results from the National Health and Nutrition Examination Surveys 2009-2014. JAMA Intern Med 2016;176:1716-7. doi:10.1001/jamainternmed. 2016.5254

14 Miller D. Maybe it's not the gluten. JAMA Intern Med 2016;176:1717-8. doi:10.1001/jamainternmed.2016.5271.

15 NPD Group. Percentage of U.S. adults trying to cut down or avoid gluten in their diets reaches new high in 2013. 2013. www.npd.com/ wps/portal/npd/us/news/press-releases/ percentage-of-us-adults-trying-to-cut-down-or-avoid-gluten-in-theirdiets-reaches-new-high-in-2013-reports-npd.

16 Estévez V, Ayala J, Vespa C, Araya M. The gluten-free basic food basket: a problem of availability, cost and nutritional composition. Eur J Clin Nutr 2016;70:1215-7. doi:10.1038/ejcn.2016.139.

17 Lee AR, Ng DL, Zivin J, Green PH. Economic burden of a gluten-free diet. I Hum Nutr Diet 2007:20:423-30. doi:10.1111/j.1365-277X.2007.00763.x

18 Burden M, Mooney PD, Blanshard RJ, White WL, Cambray-Deakin DR, Sanders DS. Cost and availability of gluten-free food in the UK: in store and online. Postgrad Med / 2015;91:622-6. doi:10.1136/ postgradmedj-2015-133395.

19 Harvard TH. Chan School of Public Health Nutrition Department. Nutrient tables. regepi.bwh.harvard.edu/health/.

20 Willett WC, Sampson L, Stampfer MJ, et al. Reproducibility and validity of a semiquantitative food frequency questionnaire. Am J Epidemiol 1985;122:51-65. doi:10.1093/oxfordjournals.aje.a114086.

21 Rimm EB, Giovannucci EL, Stampfer MJ, Colditz GA, Litin LB, Willett WC. Reproducibility and validity of an expanded self-administered semiquantitative food frequency questionnaire among male health professionals. Am J Epidemiol 1992:135:1114-26, discussion 1127-36. doi:10.1093/oxfordjournals.aje.a116211.

22 van Overbeek FM, Uil-Dieterman IG, Mol IW, Köhler-Brands L, Heymans HS, Mulder Cl. The daily gluten intake in relatives of patients with coeliac disease compared with that of the general Dutch population. Eur J Gastroenterol Hepatol 1997;9:1097-9. doi:10.1097/00042737-199711000-00013.

23 Andrén Aronsson C, Lee HS, Koletzko S, et al. TEDDY Study Group. Effects of gluten intake on risk of celiac disease: a case-control study on a Swedish birth cohort. Clin Gastroenterol Hepatol 2016;14:403409.e3. doi:10.1016/i.cgh.2015.09.030.

24 Kasarda DD. Can an increase in celiac disease be attributed to an increase in the gluten content of wheat as a consequence of wheat breeding?/ Agric Food Chem 2013;61:1155-9. doi:10.1021/ jf305122s. 
25 Qi JC, Chen JX, Wang JM, Wu FB, Cao LP, Zhang GP. Protein and hordein fraction content in barley seeds as affected by sowing date and their relations to malting quality. I Zhejiang Univ Sci B 2005;6:1069-75. doi:10.1631/jzus.2005.B1069.

26 See JA, Kaukinen K, Makharia GK, Gibson PR, Murray JA. Practical insights into gluten-free diets. Nat Rev Gastroenterol Hepatol 2015;12:580-91. doi:10.1038/nrgastro.2015.156.

27 Salvini S, Hunter DJ, Sampson L, et al. Food-based validation of a dietary questionnaire: the effects of week-to-week variation in food consumption. Int J Epidemiol 1989;18:858-67. doi:10.1093/ ije/18.4.858.

28 Feskanich D, Rimm EB, Giovannucci EL, et al. Reproducibility and validity of food intake measurements from a semiquantitative food frequency questionnaire. J Am Diet Assoc 1993;93:790-6. doi:10.1016/0002-8223(93)91754-E.

29 Yuan C, Spiegelman D, Rimm EB, et al. Validity of a dietary questionnaire assessed by comparison with multiple weighed dietary records or 24-hour recalls. Am J Epidemiol 2017;1-15.

30 Willett W, Stampfer MJ. Total energy intake: implications for epidemiologic analyses. Am J Epidemiol 1986;124:17-27. doi:10.1093/ oxfordjournals.aje.a114366.

31 Fuchs CS, Giovannucci EL, Colditz GA, et al. Dietary fiber and the risk of colorectal cancer and adenoma in women. N Engl I Med 1999:340:169-76. doi:10.1056/NEJM199901213400301.

32 Bernstein AM, Rosner BA, Willett WC. Cereal fiber and coronary hear disease: a comparison of modeling approaches for repeated dietary measurements, intermediate outcomes, and long follow-up. Eur I Epidemiol 2011;26:877-86. doi:10.1007/s10654-011-9626-x.

33 Li Y, Hruby A, Bernstein AM, et al. Saturated fats compared with unsaturated fats and sources of carbohydrates in relation to risk of coronary heart disease: a prospective cohort study. J Am Coll Cardiol 2015;66:1538-48. doi:10.1016/j.jacc.2015.07.055

34 Johnson KB, Petersen-Jones H, Thompson JM, et al. Vena cava and aortic smooth muscle cells express transglutaminases 1 and 4 in addition to transglutaminase 2. Am J Physiol Heart Circ Physiol 2012;302:H1355-66. doi:10.1152/ajpheart.00918.2011.

35 Steppan J, Sikka G, Jandu S, et al. Exercise, vascular stiffness, and tissue transglutaminase. J Am Heart Assoc 2014;3:e000599. doi:10.1161/JAHA.113.000599.

36 Ludvigsson JF, Montgomery SM, Ekbom A, Brandt L, Granath F. Small-intestinal histopathology and mortality risk in celiac disease. JAMA 2009;302:1171-8. doi:10.1001/jama.2009.1320.
37 West J, Logan RF, Card TR, Smith C, Hubbard R. Risk of vascular disease in adults with diagnosed coeliac disease: a population-based study. Aliment Pharmacol Ther 2004:20:73-9. doi:10.1111/j.1365-2036.2004.02008.x.

38 Emilsson L, Carlsson R, Holmqvist M, James S, Ludvigsson JF. The characterisation and risk factors of ischaemic heart disease in patients with coeliac disease. Aliment Pharmacol Ther 2013;37:90514. doi:10.1111/apt.12271.

39 Larsen J, Dall M, Antvorskov JC, et al. Dietary gluten increases natural killer cell cytotoxicity and cytokine secretion. Eur I Immunol 2014;44:3056-67. doi:10.1002/eji.201344264

40 Marietta EV, Gomez AM, Yeoman C, et al. Low incidence of spontaneous type 1 diabetes in non-obese diabetic mice raised on gluten-free diets is associated with changes in the intestinal microbiome. PLoS One 2013;8:e78687. doi:10.1371/journal. pone.0078687.

41 Biesiekierski JR, Newnham ED, Irving PM, et al. Gluten causes gastrointestinal symptoms in subjects without celiac disease: a double-blind randomized placebo-controlled trial. Am J Gastroenterol 2011:106:508-14, quiz 515. doi:10.1038/ajg.2010.487.

42 Biesiekierski JR, Peters SL, Newnham ED, Rosella O, Muir JG, Gibson PR. No effects of gluten in patients with self-reported non-celiac gluten sensitivity after dietary reduction of fermentable, poorly absorbed, short-chain carbohydrates. Gastroenterology 2013;145:320-8.e1, 3. doi:10.1053/j.gastro.2013.04.051

43 Vanga R, Leffler DA. Gluten sensitivity: not celiac and not certain. Gastroenterology 2013;145:276-9. doi:10.1053/j.gastro.2013.06.027.

44 Aune D, Keum N, Giovannucci E, et al. Whole grain consumption and risk of cardiovascular disease, cancer, and all cause and cause specific mortality: systematic review and dose-response metaanalysis of prospective studies. BMJ 2016;353:i2716. doi:10.1136/ bmj.i2716

45 Wu H, Flint AJ, Qi Q, et al. Association between dietary whole grain intake and risk of mortality: two large prospective studies in US men and women. JAMA Intern Med 2015;175:373-84. doi:10.1001/ jamainternmed.2014.6283.

46 Catassi C, Fabiani E, lacono G, et al. A prospective, double-blind, placebo-controlled trial to establish a safe gluten threshold for patients with celiac disease. Am J Clin Nutr 2007;85:160-6.

\section{Supplementary tables}

